\title{
Predictive Factors for Seizures and Efficacy of Antiepileptic Drugs in Patients with Aneurysmal Subarachnoid Hemorrhage
}

\author{
Ronnie E. Baticulon, ${ }^{1,2}$ Kevin Ivan P. Chan, ${ }^{2}$ Peter Paul P. Rivera, ${ }^{2}$ Gerardo D. Legaspi ${ }^{2}$ and Willy G. Lopez ${ }^{2}$ \\ ${ }^{1}$ Department of Anatomy, College of Medicine, University of the Philippines Manila \\ ${ }^{2}$ Section of Neurosurgery, Department of Neurosciences, College of Medicine and Philippine General Hospital, University of the Philippines Manila
}

\begin{abstract}
Objectives. To identify factors that predict the occurrence of seizures in patients with aneurysmal subarachnoid hemorrhage (SAH) and to evaluate the efficacy of antiepileptic drugs (AEDs) in preventing in-hospital seizures among patients who undergo clip occlusion of ruptured intracranial aneurysms.

Methods. In this retrospective study, the medical charts of 205 patients admitted for aneurysmal SAH in Philippine General Hospital (PGH) and who underwent craniotomy and clipping of aneurysm from January 2011 to June 2014 were reviewed. Demographic, radiologic, and clinical factors were converted into categorical variables and their association with the occurrence of seizures analyzed. The incidence of seizures among patients who received an AED (AED cohort) and those who did not receive an AED (No AED cohort) were compared. Secondarily, the effects of seizures and AED use on early postoperative outcomes were determined using the Glasgow Outcome Scale (GOS) on the day of discharge.
\end{abstract}

Results. Among 205 patients with aneurysmal SAH, 31 (15.1\%) developed seizures. 21 (10.2\%) had seizures at onset of SAH and only seven (3.4\%) had in-hospital seizures. Aneurysm re-rupture (OR 5.26, p-value 0.045) and the presence of a parenchymal clot (OR 2.90, p-value 0.043) were independent predictors for seizure occurrence. There was no significant difference in the incidence of seizures in the AED cohort and in the No AED cohort (4/100, $4 \%$ vs. $3 / 99,3 \%$, p-value 0.714$)$. AED use was associated with a higher proportion of patients with a discharge GOS score of 3 or less (28.0\% vs $12.1 \%$, p-value 0.005$)$.

Conclusion. The results of the study do not support the routine use of AEDs in patients with aneurysmal SAH.

Key Words: seizure, aneurysm, subarachnoid hemorrhage, craniotomy, phenytoin, levetiracetam, antiepileptic drugs

\section{INTRODUCTION}

Corresponding author: Ronnie E. Baticulon, MD Department of Anatomy

College of Medicine

University of the Philippines Manila

547 Pedro Gil St., Ermita, Manila 1000, Philippines

Email: rebaticulon@up.edu.ph
Seizures are common in patients with aneurysmal subarachnoid hemorrhage (SAH), with an incidence ranging from six to $21 \%$ in different studies. ${ }^{1-7}$ Of the 1,070 patients who underwent clip occlusion in the International Subarachnoid Aneurysm Trial (ISAT), 146 (13.6\%) developed a seizure. ${ }^{8}$ Most seizures occur at the onset of $\mathrm{SAH}$, during the first 24 hours after ictus, or after a rebleed. ${ }^{7,9,10}$ A systematic review of 25 studies has shown that postoperative seizures develop early (i.e., in-hospital) in $2.3 \%$ of patients, while $5.5 \%$ get a seizure after discharge from the hospital. ${ }^{11}$ As high as 7\% of patients with $\mathrm{SAH}$ will develop epilepsy later on..$^{5,12}$

Previously identified factors that increase the risk of seizures after aneurysmal SAH include poor neurologic grade on admission, greater subarachnoid clot burden, 
subdural hematoma, intraventricular hemorrhage, delayed ischemic neurologic deficit due to vasospasm, thromboembolic complications, and a middle cerebral artery aneurysm location. ${ }^{2,3,7}$

It remains debatable whether the occurrence of perioperative seizures is an independent predictor of poor outcome. In a review of $527 \mathrm{SAH}$ patients, although seizure occurrence was associated with poor functional outcome at 14 days, it was not a significant factor contributing to poor outcome in the long term. ${ }^{13}$ In contrast, onset seizure following aneurysmal and non-aneurysmal $\mathrm{SAH}$ has been shown to be an independent predictor of disability six weeks post-SAH, ${ }^{14}$ while seizure burden even when nonconvulsive - was associated with poorer functional and cognitive outcome at three months. ${ }^{15}$

There are no consensus recommendations on when to start antiepileptic drugs (AEDs) for aneurysmal $\mathrm{SAH}$, which drug to use, at what dose, and for how long. Prescribing behavior for AEDs varies across countries and neurovascular centers. The choice and duration of AED therapy for primary and secondary prevention of seizures in SAH are not agreed upon. ${ }^{4,16-21}$

On analysis of data collected from 3552 patients enrolled in four clinical trials between 1991 and 1997, 65.1\% received at least one AED. However, the rate of AED use ranged from seven to $93 \%$, depending on which country. Patients who were young, with a worse neurologic grade, and with lower systolic blood pressure on admission were more likely to be started on AED therapy. ${ }^{10}$ A 2013 Cochrane review has shown that there are still no randomized controlled trials that assess the efficacy and safety of AEDs compared to placebo in the primary and secondary prevention of seizures after $\mathrm{SAH}$, precluding any recommendation. ${ }^{22}$ Even the recommendations in the 2012 American Heart Association/ American Stroke Association guideline on the management of aneurysmal SAH are vague, saying that AEDs "may be considered in the immediate post-hemorrhagic period."

However, it is imperative that clear guidelines be established given the emergent and life-threatening nature of SAH following aneurysm rupture. The annual incidence of aneurysmal SAH in the Western Pacific region has been estimated to be 12.38 per 100,000 persons, ${ }^{23}$ which translates to an annual burden of approximately 13,000 Filipino patients each year.

In Philippine General Hospital (PGH), a tertiary government hospital which serves as the national university hospital, charity patients admitted for aneurysmal SAH are managed primarily by craniotomy and clipping of the ruptured aneurysm. These are public patients whose health care expenses are subsidized by the government. Owing mainly to financial constraints and delayed consult or transfer, many patients are unable to undergo immediate surgery.

It has been postulated that the delay in intervention may put these patients at an increased risk of developing seizures, thereby warranting the use of AEDs, even in patients with no seizure at onset of SAH. Just a single seizure may potentially trigger re-bleeding and subsequently lead to secondary brain injury. ${ }^{6}$

Any potential benefit of seizure prophylaxis has to be weighed against possible adverse effects from AED use. Phenytoin, for instance, is known to cause fever and rash, but serious complications such as Stevens-Johnson syndrome, liver failure, and pancreatitis have also been documented. ${ }^{17}$ It may also interfere with the metabolism of other drugs through the induction of the CYP 450 enzyme. ${ }^{18}$ Its use has been correlated, in a dose-dependent manner, with cognitive disability in patients with aneurysmal SAH at 14 days and three months after ictus. ${ }^{13}$ Even prophylactic levetiracetam has been associated with a higher rate of delayed ischemic neurologic deficits in patients with SAH. ${ }^{7}$

The results of this retrospective study would aid in the development of a protocol on AED therapy for primary and secondary prevention of seizures in patients with aneurysmal SAH, especially in a resource-limited setting.

\section{METHODS}

This is a retrospective cohort study of charity patients admitted for aneurysmal SAH who underwent craniotomy and clipping of aneurysm in PGH for a period of 42 months from January 2011 to June 2014. Ethics approval was obtained from the University of the Philippines Manila Research Ethics Board (Registration No. NEU 2014-25-01).

A total of 233 consecutive patients were identified to be eligible for inclusion in the study. The charts of 210 (90.1\%) patients were available for review and retrieved from the Medical Records Section of PGH. Additional clinical data, when available, were obtained from the weekly vascular census of the neurosurgery service. Five patients had to be excluded because their in-patient charts were incomplete and lacked information critical to the study.

The 205 patients were divided into two groups: those who were started on AED therapy at any point from admission to discharge (AED cohort) and those who did not receive therapy (No AED cohort). If a patient developed an in-hospital seizure and was not on an AED at that time, data of the patient were analyzed with the No AED cohort.

In the final quantitative analysis, six patients had to be excluded from the AED cohort because either phenytoin $(n=5)$ or phenobarbital $(n=1)$ was given for three days or less, without a loading dose, and in so doing, these drugs would not have reached therapeutic levels in the serum.

For both cohorts, all patient data were anonymized and relevant demographic, clinical, and radiologic information were encoded in standardized data collection forms. Collected data included age, sex, presence of hypertension, history of smoking, Hunt and Hess $(\mathrm{HH})$ grade on admission, imaging findings, location of ruptured aneurysm, 
interval from onset of SAH to admission and surgery, occurrence of re-bleeding, clinical or radiologic evidence of vasospasm, and length of hospital stay.

Information regarding the presence of a localized clot, parenchymal hematoma, or intraventricular hemorrhage on computed tomography (CT) or magnetic resonance imaging (MRI) was obtained from the clinical notes of attending physicians.

A Glasgow Outcome Scale (GOS) score was given for each patient based on the patient's neurologic examination and functional status on the day of discharge. A GOS score of 5 (good recovery) or 4 (moderate disability) was considered a favorable outcome while a score of 3 or less (severe disability, vegetative state, dead) was recorded as an unfavorable outcome.

As used previously by Lin and coworkers in their paper that analyzed perioperative seizures and epilepsy following aneurysmal $\mathrm{SAH},{ }^{5}$ seizure was defined as repetitive, rhythmic jerking, with or without preceding tonic spasms, that was focal or generalized in nature, with or without loss of consciousness; vasospasm was defined as development of focal neurological signs or deterioration in conscious state with either an unchanged CT scan or evidence of cerebral infarction with no other discernible cause, and re-bleeding was defined as sudden deterioration of the clinical state accompanied by new or increased blood on cerebral CT scans.

The occurrence of seizures was extracted from the patient records and the incidence of seizures calculated in each cohort. In-hospital seizures were classified as: preoperative seizures, defined as those occurring from the time of admission until surgery; or early postoperative seizures, defined as those occurring from the time of surgery until discharge from hospital.

Seizures that occurred from the onset of SAH until just before hospital admission, as documented in the patient's clinical history, were included in the calculation of overall seizure incidence. When clinical information was available, the seizures were characterized. Relevant data such as laboratory parameters or uncontrolled co-morbid conditions at the time of seizure episodes were also noted.

The proportion of patients who developed seizures and the mean duration of hospital stay (in days) were computed in the AED and No AED cohorts. The GOS scores were tabulated. The difference between both groups was analyzed for statistical significance at $95 \%$ confidence interval using t-test or $\chi^{2}$ test, as appropriate.

In addition, clinical data were converted into categorical variables and the relationship between variables and the occurrence of seizures analyzed for statistical significance, using the $\mathrm{t}$-test or $\chi^{2}$ test, as appropriate, at $95 \%$ confidence interval. In all analyses, a p-value of $\leq$ 0.05 was considered significant. Multivariate regression analysis was then performed to determine which clinical data were independent predictors of seizure occurrence among patients with aneurysmal SAH.
All data were encoded in Microsoft Excel 2007 and all statistical analyses were performed using GraphPad Prism version 6.0f for Mac OS X.

\section{RESULTS}

\section{Clinical Data and Outcomes of Aneurysm Clipping in PGH}

The characteristics of the study population are shown in Table 1. Of the 205 patients included in this study, 130 (63.4\%) were females. The mean age was $50.1 \pm 9.6$ years old (range: 23-72). 173 (84.4\%) had hypertension and 78 (38.6\%) were current or previous smokers. No patient had preexisting epilepsy or had been taking AEDs prior to seeking medical attention for SAH. 200 patients (97.6\%) were previously seen at a local clinic or hospital, before being transferred to PGH for definitive management.

The median duration from ictus until admission was five days (mean: 10.5 \pm 16.0 days, range: $0-121$ days, date of ictus unknown in three patients). 156 patients (76.0\%) were either HH I or II on admission. Median duration from ictus to surgery was 13.5 days (mean: 19.6 \pm 18.7 days, range: $2-125$ days). Only 19 patients (9.3\%) underwent surgery within seven days from ictus and only 113 patients (55.1\%) within 14 days.

The most common location of the ruptured aneurysm was the anterior communicating artery (77, 37.6\%), followed by the middle cerebral artery $(54,26.3 \%)$. Multiple aneurysms were documented in 29 patients (14.1\%), and two or more aneurysms were clipped on the same sitting in 14 of them. Two patients also had an arteriovenous malformation.

Attendant hydrocephalus had to be surgically treated in nine patients: four underwent external ventricular drainage prior to clip occlusion while five underwent ventriculoperitoneal shunt insertion as a second operation. Re-bleeding occurred in 11 patients (5.4\%): prior to admission in two patients and while admitted in the remaining nine.

Seizures developed in 31/205 patients (15.1\%). These occurred at the onset of SAH in 21 patients (10.2\%) and recurred at least once in seven patients (3.4\%).

Overall, a total of 42 seizures were documented. Twenty-six seizures $(61.9 \%)$ were prior to admission at any health care facility, $11(26.2 \%)$ were preoperative, and five $(11.9 \%)$ were early postoperative. Twenty-six seizures (61.9\%) occurred within seven days of ictus, with an additional 8 (19.0\%) developing from day 8 until day 30 post-SAH. Thirty-one seizures $(73.8 \%)$ were generalized, six $(14.3 \%)$ were focal, and five $(11.9 \%)$ were insufficiently described precluding classification.

Only seven out of 205 patients (3.4\%) had in-hospital seizures. In five of them, the seizure was associated with aneurysm re-rupture. One patient had a postoperative intraventricular hemorrhage and the last patient had hyponatremia (serum Na: $120 \mathrm{mEq} / \mathrm{L}$ ) and was septic from pneumonia and urinary tract infection. 
Table 1. Clinical Data and Outcomes of Aneurysm Clipping in $\mathrm{PGH}$

\begin{tabular}{|c|c|}
\hline Variable & No. (\%) \\
\hline $\begin{array}{l}\text { Male } \\
\text { Female }\end{array}$ & $\begin{array}{c}75(36.6 \%) \\
130(63.4 \%)\end{array}$ \\
\hline Age $^{\neq}$ & $\begin{array}{c}50.1 \pm 9.6 \\
\text { (Range: } 23-72 \text { ) }\end{array}$ \\
\hline Hypertension present & $173(84.4 \%)$ \\
\hline Current or previous smoker $\neq$ & $78(38.6 \%)$ \\
\hline $\begin{array}{l}\text { Hunt and Hess Grade } \\
\text { I } \\
\text { II } \\
\text { III } \\
\text { IV } \\
\text { V } \\
\end{array}$ & $\begin{array}{c}97(47.3 \%) \\
59(28.8 \%) \\
37(18.0 \%) \\
12(5.9 \%) \\
0(0 \%) \\
\end{array}$ \\
\hline $\begin{array}{l}\text { Location of Ruptured Aneurysm } \\
\text { AComm } \\
\text { MCA } \\
\text { ICA } \\
\text { ACA } \\
\text { PComm } \\
\text { BA } \\
\text { AChA } \\
\text { PCA } \\
\text { PICA } \\
\text { SCA }\end{array}$ & $\begin{array}{c}77(37.6 \%) \\
54(26.3 \%) \\
34(16.6 \%) \\
19(9.3 \%) \\
13(6.3 \%) \\
4(2.0 \%) \\
1(0.5 \%) \\
1(0.5 \%) \\
1(0.5 \%) \\
1(0.5 \%) \\
\end{array}$ \\
\hline $\begin{array}{l}\text { Seizures } \\
\text { Any seizure } \\
\text { At onset of SAH } \\
\text { In-hospital seizures } \\
\text { Never }\end{array}$ & $\begin{array}{c}31(15.1 \%) \\
21(10.2 \%) \\
7(3.4 \%) \\
174(84.9 \%)\end{array}$ \\
\hline $\begin{array}{l}\text { GOS score on discharge } \\
5 \text { - Good recovery } \\
4 \text { - Moderate disability } \\
3 \text { - Severe disability } \\
2 \text { - Vegetative state } \\
1 \text { - Dead }\end{array}$ & $\begin{array}{c}133(64.9 \%) \\
32(15.6 \%) \\
29(14.1 \%) \\
2(1.0 \%) \\
9(4.4 \%) \\
\end{array}$ \\
\hline Median duration from ictus to admission ${ }^{\neq \# \neq}$ & $\begin{array}{c}5 \text { days } \\
\text { Mean: } 10.5 \pm 16.0 \\
\text { Range: } 0-121 \text { days } \\
\end{array}$ \\
\hline Median duration from ictus to surgery & $\begin{array}{c}13.5 \text { days } \\
\text { Mean: } 19.6 \pm 18.7 \\
\text { Range: } 2-125 \text { days } \\
\end{array}$ \\
\hline Duration of hospital stay ${ }^{\ddagger}$ & $\begin{array}{c}18.0 \pm 11.2 \text { days } \\
\text { Range: } 7-105 \text { days }\end{array}$ \\
\hline Preoperative length of stay ${ }^{\ddagger}$ & $\begin{array}{c}9.2 \pm 8.5 \text { days } \\
\text { Range: } 0-82 \text { days }\end{array}$ \\
\hline Postoperative length of stay ${ }^{\ddagger}$ & $\begin{array}{c}7.8 \pm 6.3 \text { days } \\
\text { Range: } 3-44 \text { days }\end{array}$ \\
\hline $\begin{array}{l}n=205 \text {, unless otherwise indicated } \\
\neq \text { Values expressed as the mean } \pm \text { standard } d \\
\text { \#f Smoking history reported in } 202 \text { patients } \\
\neq \neq \neq \text { Exact date of ictus known in } 202 \text { patients } \\
\text { AComm - Anterior communicating artery } \\
\text { MCA - Middle cerebral artery } \\
\text { ICA - Internal carotid artery } \\
\text { ACA - Anterior cerebral artery } \\
\text { PComm - Posterior communicating artery } \\
\text { BA - Basilar artery } \\
\text { AChA - Anterior choroidal artery } \\
\text { PCA - Posterior cerebral artery } \\
\text { PICA - Posterior inferior cerebellar artery } \\
\text { SCA - Superior cerebellar artery } \\
\text { SAH - Subarachnoid hemorrhage }\end{array}$ & \\
\hline
\end{tabular}

Table 2. Risk Factors for Seizures Following Subarachnoid Hemorrhage

\begin{tabular}{lccc}
\multicolumn{1}{c}{ Variables } & $\begin{array}{l}\text { Odds } \\
\text { Ratio }\end{array}$ & Pvalue & 95\% Cl \\
\hline$\geq 60$ years vs. 19 to 59 years & 0.32 & 0.182 & $0.06-1.70$ \\
Male vs. Female & 1.95 & 0.273 & $0.59-6.47$ \\
Hypertension & 1.40 & 0.628 & $0.36-5.46$ \\
Smoking & 0.92 & 0.888 & $0.29-2.88$ \\
HH grade III-IV vs. I-II & 2.02 & 0.178 & $0.72-5.67$ \\
Localized clot or parenchymal hematoma & 2.90 & 0.043 & $1.03-8.11$ \\
Intraventricular hemorrhage & 2.26 & 0.117 & $0.81-6.29$ \\
Location of ruptured aneurysm & 1.66 & 0.247 & $0.70-3.94$ \\
Vasospasm & 0.57 & 0.297 & $0.20-1.62$ \\
Re-bleeding & 5.26 & 0.045 & $1.04-26.63$ \\
Timing of surgery, early vs. late & 1.04 & 0.956 & $0.22-4.82$ \\
\hline
\end{tabular}

Univariate analyses showed that a patient was more likely to have seizures when any of the following factors were present: HH III or IV (Odds Ratio, OR: 2.03), intraventricular hemorrhage (OR: 2.29), localized clot or parenchymal hematoma on imaging (OR: 2.95), aneurysm in the posterior circulation (OR: 8.44), and re-rupture of aneurysm (OR: 5.39). Age, sex, presence of hypertension, smoking history, presence of vasospasm, and timing of surgery (early, $\leq 7$ days post-ictus vs. late, $>7$ days post-ictus) had no influence on the development of seizures following SAH.

However, when logistic regression analysis was performed (Table 2), only the presence of a localized clot or parenchymal hematoma ( $\mathrm{p}$-value 0.043 ) and the occurrence of re-bleeding ( $\mathrm{p}$-value 0.045 ) were found to be significant independent predictors of increased seizure risk.

A hundred and sixty-five patients (80.1\%) had a GOS score of either 5 or 4 on the day of discharge. Nine out of 205 patients died during their hospital admission, resulting in an in-hospital mortality rate of $4.4 \%$ for this series. The causes of death were acute myocardial infarction $(n=1)$, septic shock $(n=3)$, and brain herniation $(n=5)$ from hydrocephalus, diffuse cerebral edema, or infarction.

\section{Efficacy of AEDs in Prevention of Seizures}

There were more males in the No AED cohort ( $42 \%$ vs. 29\%, p-value 0.048). On the other hand, the AED cohort included a significantly higher number of patients who were HH III or IV on admission ( $36 \%$ vs. $13 \%$, p-value 0.0002) and who had a localized clot or parenchymal hematoma on CT (28\% vs. $13 \%$, p-value 0.020). Aside from these, the two cohorts were identical in the other variables tested: age, presence of hypertension, smoking history, location of a ruptured aneurysm, intraventricular hemorrhage, vasospasm, and re-bleeding. (Table 3)

Four of the patients who had in-hospital seizures belonged to the AED cohort, while 3 belonged to the No AED cohort. The marginal difference in the risk of developing in-hospital seizures between the two cohorts $(4 / 100,4.0 \%$ vs. $3 / 99,3.0 \%)$ was not statistically significant ( $p$-value 
Table 3. Comparison Between AED and No AED Cohorts

\begin{tabular}{|c|c|c|c|}
\hline & AED Cohort & No AED Cohort & P value \\
\hline Male & $29 / 100(29 \%)$ & $42 / 99(42 \%)$ & 0.048 \\
\hline Age, mean \pm SD & $50.0 \pm 10.0$ & $50.4 \pm 9.1$ & 0.742 \\
\hline Hypertension present & $86 / 100(86 \%)$ & $83 / 99(84 \%)$ & 0.670 \\
\hline Current or previous smoker ${ }^{\ddagger}$ & $39 / 100(39 \%)$ & $35 / 96(36 \%)$ & 0.714 \\
\hline $\mathrm{HH}$ grade III and IV & $36 / 100(36 \%)$ & $13 / 99(13 \%)$ & 0.0002 \\
\hline $\begin{array}{l}\text { Aneurysm Location } \\
\text { ACA } \\
\text { AComm } \\
\text { MCA } \\
\text { ICA } \\
\text { PComm/AChA } \\
\text { BA/PCA/PICA/SCA }\end{array}$ & $\begin{array}{c}9(9 \%) \\
32(32 \%) \\
30(30 \%) \\
17(17 \%) \\
8(8 \%) \\
4(4 \%)\end{array}$ & $\begin{array}{c}8(8 \%) \\
42(42 \%) \\
23(23 \%) \\
17(17 \%) \\
6(6 \%) \\
3(3 \%)\end{array}$ & 0.737 \\
\hline Localized clot or parenchymal hematoma ${ }^{\neq \neq}$ & $25 / 88(28 \%)$ & $9 / 69(13 \%)$ & 0.020 \\
\hline Intraventricular hemorrhage $\mathrm{f}^{\ddagger \ddagger}$ & $25 / 88(28 \%)$ & $17 / 69(25 \%)$ & 0.596 \\
\hline Vasospasm & $30 / 100(30 \%)$ & $27 / 99(27 \%)$ & 0.671 \\
\hline Re-bleeding & $8 / 100(8 \%)$ & $3 / 99(3 \%)$ & 0.125 \\
\hline Incidence of in-hospital seizures & $4 / 100(4.0 \%)$ & $3 / 99(3.0 \%)$ & 0.714 \\
\hline
\end{tabular}

Table 4. AED Use and Early Postoperative Outcome

\begin{tabular}{cccc} 
& AED Cohort & No AED Cohort & P value \\
HH grade I to IV & & & \\
GOS 5 or 4 & $72(72.0 \%)$ & $87(87.9 \%)$ & 0.005 \\
GOS 3 or less & $28(28.0 \%)$ & $12(12.1 \%)$ & \\
HH grade I and II only & & & \\
GOS 5 or 4 & $51(79.7 \%)$ & $79(91.9 \%)$ & 0.030 \\
GOS 3 or less & $13(20.3 \%)$ & $7(8.1 \%)$ & \\
\hline
\end{tabular}

0.714). Of note, none of the 6 patients excluded because of subtherapeutic AED dosing developed a seizure.

Thus, a total of 109 patients from both cohorts received an AED, regardless of dose or duration (NB: the three patients in the No AED cohort were given AEDs after they developed in-hospital seizures). In 79 patients (38.5\%), the AED was started on admission. The indication for AED therapy as the primary prevention of seizures in 84 patients and secondary prevention in the remaining 25 patients.

The treatment regimen was monotherapy in almost all patients (97.2\%), with only three patients requiring a second-line, add-on drug for seizure control. The mean duration of AED use was $14.3 \pm 12.6$ days (range: $1-103$ ). The most commonly prescribed first-line AEDs were levetiracetam (76.1\%) and phenytoin (22.9\%). In 2011, a patient started on an AED was just as likely to receive phenytoin (19/40, 47.5\%) as levetiracetam (20/40, 50\%). But from 2012 to 2014, only five patients received phenytoin compared to 63 who received levetiracetam. 48 patients (23.4\%) were previously given at least one dose of an AED prior to being transferred to the study center.

In 1562 patient-hospital days, only one adverse event probably related to AED use was reported: a $55 / \mathrm{F}$ $\mathrm{HH}$ III patient who developed episodes of agitation and hallucination after having received five doses of levetiracetam at $500 \mathrm{mg}$ twice daily. The AED was then shifted to carbamazepine. 66 patients (32.2\%) were eventually discharged with an AED included in their home medications.

Excluding patients who died and patients who received subtherapeutic doses of AEDs, patients were given an AED tended to have a longer duration of hospital stay compared with patients who were not given an AED (19.4 days vs. 16.2 days, p-value 0.020). However, when analysis was limited to HH I and II patients (i.e., good clinical grade), the mean duration of hospital stay of both patient groups had no significant difference (16.9 days vs. 15.4 days, $\mathrm{p}$-value 0.261 ).

Initiation of AED therapy was also associated with a higher number of patients with unfavorable outcomes: $28.0 \%$ in those who received an AED vs. $12.1 \%$ in those who did not ( $p$-value 0.005 ). In contrast to the hospital stay, the difference in the proportion of unfavorable outcomes remained statistically significant, even if the analysis were limited to HH I and II patients (20.3\% vs $8.1 \%$, p-value 0.030).(Table 4)

The occurrence of seizures at any point after aneurysmal $\mathrm{SAH}$ had no effect on the early postoperative outcome of patients who underwent clip occlusion. In those who had at least one seizure - whether pre-hospital or perioperative 
- the proportion of patients with unfavorable GOS scores was not significantly different from the proportion of patients with unfavorable outcomes in those who never had a seizure (9/31 patients, $29.0 \%$ vs. $31 / 174$ patients, $17.8 \%$, $\mathrm{p}$-value 0.147$)$. It should be noted that six of seven patients who developed in-hospital seizures either died or had severe disability at time of discharge from the hospital.

\section{DISCUSSION}

The calculated incidence and observed the timing of seizures in this study are comparable with data previously reported in the literature. Most seizures occurred at onset or prior to seeking medical attention; thus, these seizures may be considered unpreventable. A caveat is that a real seizure could not be reliably differentiated from seizure-like movements (e.g., decorticate posturing from acutely elevated increased intracranial pressure after $\mathrm{SAH}$ ) when relying only on eyewitness accounts of non-medical bystanders. ${ }^{5}$

The results of the current study do not support routine use of AEDs in all patients with aneurysmal SAH. The incidence of in-hospital seizures did not significantly differ, with or without AED use, at $4 \%$ and $3 \%$, respectively. Retrospective studies in Korea and North America showed similar results. ${ }^{7,24}$ In the latter, propensity score matched-analysis of 353 patients showed that the rate of seizure occurrence was the same for patients who received prophylactic AEDs and those who did not. These findings are particularly important in low- or middle-income countries where a common reason for the delay in definitive treatment (i.e., clipping of aneurysm) is the lack of resources for diagnostic procedures and surgery. In this situation, it might be more prudent to prioritize the acquisition of an angiogram or procurement of an aneurysm clip, over the initiation and maintenance of AEDs, all of which entail an economic cost.

AED use was associated with worse early postoperative outcomes in this retrospective review, with a higher incidence of patients who died during admission and who had severe disability or were in a vegetative state on the day of discharge. This echoes the findings of Rosengart's study that pooled data from 3,552 patients and showed that prophylactic AED use was associated with increased inhospital complications and worse GOS scores. ${ }^{10}$

Studies that link AED use to poor outcomes are often criticized for selection bias, citing that attending physicians are more likely to prescribe AEDs in patients with more severe disease, and who thus have an inherently worse prognosis. ${ }^{9}$ This is the proposed explanation for the longer mean duration of hospital stay of patients in the AED cohort. Rather than a direct relationship with AED use, the figures reflect the cohort's significantly higher number of HH III or IV patients, whose postoperative care necessarily takes longer and who are more likely to suffer from significant medical co-morbidities or develop postoperative complications.
The figures also indicate that attending physicians are more likely to start seizure prophylaxis in patients who are admitted with impairment in sensorium or more severe neurologic deficits. True enough, when subgroup analysis limiting to HH I and II patients is performed, there is no significant difference in the length of stay of patients in the AED and No AED groups.

The same cannot be said for the relationship of AED use with early postoperative outcomes. Even when the outcomes were adjusted for $\mathrm{HH}$ grade on admission, the patients who received an AED fared worse than their counterparts who did not receive an AED. Still, this finding should not be deemed conclusive, considering that although the two cohorts are similar, the patients have not been matched in all possible criteria that may affect the outcome of treatment after aneurysmal SAH. The heterogeneity of the AED regimens used in this population also precluded further analyses to determine if the association is dependent on the choice of AED or dose used.

The occurrence of seizures after aneurysmal SAH did not necessarily correlate with an unfavorable early postoperative outcome. The likelihood of getting a GOS score of 5 or 4 on discharge was similar in patients who had at least one seizure episode and those who never had any. The unfavorable outcomes seen in six of seven patients who developed an in-hospital seizure should be interpreted with caution, especially since in five of them, the seizure was associated with a re-bleed, and in the other two patients, significant co-morbid conditions were present, all of which portend a poorer prognosis in patients with $\mathrm{SAH}$. It would be more prudent to consider the seizures as indicators of the patients' worsening neurologic status.

\section{Limitations}

Because PGH has yet to implement a protocol on AED use for SAH patients, there was no standard AED regimen used in this study. The choice of AED including dose, timing, and duration - was largely arbitrary and dependent on the attending physician.

Also, since a majority of patients were previously managed in another clinic or hospital, the authors had limited reliable and objective data on AED use prior to being admitted to the study center. There were 15 patients who received an AED prior to transfer but were not started on AEDs on admission to PGH, hence they were included in the No AED cohort. None of these 15 patients developed an in-hospital seizure. Notwithstanding, even if this subset of patients were regrouped into the AED cohort (assuming that all of them received adequate seizure prophylaxis or treatment), there would still be no beneficial effect with AED use. The difference in seizure risk remained statistically insignificant (3.4\% AED vs. 3.6\% No AED, p-value 0.972). The authors believe that the current study design mirrors what a neurologist or neurosurgeon would encounter in clinical practice: deciding whether to initiate AEDs or not 
in a patient with aneurysmal SAH, who may or may not have received AEDs from another hospital.

A retrospective study design also has its inherent limitations. For instance, the rate of adverse effects from AED use is likely an underestimate because the attending physicians may have failed to document all possible drug effects in the medical charts. Further, because the authors did not have outpatient follow-up data for patients included in this study, it is not known whether the observed differences in outcomes persisted in the long term.

\section{CONCLUSION}

In this retrospective study of 205 patients who underwent clip occlusion of ruptured aneurysms in a tertiary hospital, initiation of AED therapy had no effect on the rate of occurrence of seizures after aneurysmal SAH. Aneurysm re-rupture and the presence of a parenchymal clot or localized hematoma were found to be independent predictors for the development of seizures following aneurysmal SAH. A prospective randomized clinical trial with long-term follow up is recommended to formulate definitive guidelines on AED use after aneurysmal SAH.

\section{Acknowledgment}

The authors would like to acknowledge Dr. John Carlo Reyes of the University of the Philippines College of Medicine for his invaluable assistance in the statistical analyses of data used in the study.

\section{Statement of Authorship}

All authors conceptualized and designed the study. REB and KPC collected the data. REB performed data analysis and drafted the manuscript. All authors revised and approved the final version submitted.

\section{Author Disclosure}

All authors declared no conflicts of interest.

\section{Funding Source}

This paper was funded by the primary investigator.

\section{REFERENCES}

1. Connolly ES Jr, Rabinstein AA, Carhuapoma JR, Derdeyn CP, Dion J, Higashida RT, et al. Guidelines for the management of aneurysmal subarachnoid hemorrhage: a guideline for healthcare professionals from the American Heart Association/ american Stroke Association. Stroke 2012; 43(6):1711-37.

2. Ibrahim GM, Fallah A, Macdonald RL. Clinical, laboratory, and radiographic predictors of the occurrence of seizures following aneurysmal subarachnoid hemorrhage. J Neurosurg. 2013;119(2):347-52.

3. Hart Y, Sneade M, Birks J, Rischmiller J, Kerr R, Molyneux A. Epilepsy after subarachnoid hemorrhage: the frequency of seizures after clip occlusion or coil embolization of a ruptured cerebral aneurysm: results from the International Subarachnoid Aneurysm Trial. J Neurosurg. 2011;115(6):1159-68.
4. Choi KS, Chun HJ, Yi HJ, Ko Y, Kim YS, Kim JM. Seizures and epilepsy following aneurysmal subarachnoid hemorrhage: incidence and risk factors. J Korean Neurosurg Soc. 2009; 46(2):93-8.

5. Lin CL, Dumont AS, Lieu AS, Yen CP, Hwang SL, Kwan AL, et al. Characterization of perioperative seizures and epilepsy following aneurysmal subarachnoid hemorrhage. J Neurosurg. 2003; 99(6):978-85.

6. Lin YJ, Chang WN, Chang HW, Ho JT, Lee TC, Wang HC, et al. Risk factors and outcome of seizures after spontaneous aneurysmal subarachnoid hemorrhage. Eur J Neurol. 2008; 15(5):451-7.

7. Panczykowski D, Pease M, Zhao Y, Weiner G, Ares W, Crago E, et al. Prophylactic antiepileptics and seizure incidence following subarachnoid hemorrhage: a propensity score-matched analysis. Stroke. 2016; 47(7):1754-60.

8. Molyneux AJ, Kerr RS, Yu LM, Clarke M, Sneade M, Yarnold $\mathrm{JA}$, et al. International subarachnoid aneurysm trial (ISAT) of neurosurgical clipping versus endovascular coiling in 2143 patients with ruptured intracranial aneurysms: a randomised comparison of effects on survival, dependency, seizures, rebleeding, subgroups, and aneurysm occlusion. Lancet. 2005; 366(9488):809-17.

9. Lanzino G, D'Urso PI, Suarez J, Participants in the International Multi-disciplinary Consensus Conference on the Critical Care Management of Subarachnoid Hemorrhage. Seizures and anticonvulsants after aneurysmal subarachnoid hemorrhage. Neurocrit Care. 2011; 15(2):247-56.

10. Rosengart AJ, Huo JD, Tolentino J, Novakovic RL, Frank JI, Goldenberg FD, et al. Outcome in patients with subarachnoid hemorrhage treated with antiepileptic drugs. J Neurosurg. 2007; 107(2):253-60.

11. Raper DM, Starke RM, Komotar RJ, Allan R, Connolly ES Jr. Seizures after aneurysmal subarachnoid hemorrhage: a systematic review of outcomes. World Neurosurg. 2013; 79(5-6):682-90.

12. Claassen J, Peery S, Kreiter KT, Hirsch LJ, Du EY, Connolly ES, et al. Predictors and clinical impact of epilepsy after subarachnoid hemorrhage. Neurology. 2003; 60(2):208-14.

13. Naidech AM, Kreiter KT, Janjua N, Ostapkovich N, Parra A, Commichau $\mathrm{C}$, et al. Phenytoin exposure is associated with functional and cognitive disability after subarachnoid hemorrhage. Stroke. 2005; 36(3):583-7.

14. Butzkueven H, Evans AH, Pitman A, Leopold C, Jolley DJ, Kaye $\mathrm{AH}$, et al. Onset seizures independently predict poor outcome after subarachnoid hemorrhage. Neurology. 2000;55(9):1315-20.

15. De Marchis GM, Pugin D, Meyers E, Velasquez A, Suwatcharangkoon S, Park S, et al. Seizure burden in subarachnoid hemorrhage associated with functional and cognitive outcome. Neurology. 2016; 86(3):253-60.

16. Dewan MC, Mocco J. Current practice regarding seizure prophylaxis in aneurysmal subarachnoid hemorrhage across academic centers. J Neurointerv Surg. 2015; 7(2):146-9.

17. Chumnanvej S, Dunn IF, Kim DH. Three-day phenytoin prophylaxis is adequate after subarachnoid hemorrhage. Neurosurgery. 2007; 60(1):99-102-discussion102-3.

18. Murphy-Human T, Welch E, Zipfel G, Diringer MN, Dhar R. Comparison of short-duration levetiracetam with extended-course phenytoin for seizure prophylaxis after subarachnoid hemorrhage. World Neurosurg. 2011; 75(2):269-74.

19. Rhoney DH, Tipps LB, Murry KR, Basham MC, Michael DB, Coplin WM. Anticonvulsant prophylaxis and timing of seizures after aneurysmal subarachnoid hemorrhage. Neurology. 2000;55(2):258-65.

20. Kodankandath TV, Farooq S, Wazni W, Cox J, Southwood C, Rozansky G, et al. Seizure prophylaxis in the immediate posthemorrhagic period in patients with aneurysmal subarachnoid hemorrhage. J Vasc Interv Neurol. 2017; 9(6):1-4.

21. Human T, Diringer MN, Allen M, Zipfel GJ, Chicoine M, Dacey R, et al. A Randomized Trial of Brief Versus Extended Seizure Prophylaxis After Aneurysmal Subarachnoid Hemorrhage. Neurocrit Care. 2018; 28(2):169-74. 
22. Marigold R, Günther A, Tiwari D, Kwan J. Antiepileptic drugs for the primary and secondary prevention of seizures after subarachnoid haemorrhage. Cochrane Database Syst Rev. 2013; (6):CD008710.

23. Hughes JD, Bond KM, Mekary RA, Dewan MC, Rattani A, Baticulon R, et al. Estimating the global incidence of aneurysmal subarachnoid hemorrhage: a systematic review for central nervous system vascular lesions and meta-analysis of ruptured aneurysms. World Neurosurg. 2018; 115:430-47.

24. Yoon SJ, Joo JY, Kim YB, Hong CK, Chung J. Effects of prophylactic antiepileptic drugs on clinical outcomes in patients with a good clinical grade suffering from aneurysmal subarachnoid hemorrhage. J Cerebrovasc Endovasc Neurosurg. 2015; 17(3):166-72.
25. Hunt WE, Hess RM. Surgical risk as related to time of intervention in the repair of intracranial aneurysms. J Neurosurg. 1968; 28(1):14-20.

26. Jennett B, Snoek J, Bond MR, Brooks N. Disability after severe head injury: observations on the use of the Glasgow Outcome Scale. J Neurol Neurosurg Psychiatry. 1981; 44(4):285-93.

\section{APPENDICES}

Appendix A.1. Hunt and Hess Grading for Aneurysmal Subarachnoid Hemorrhage ${ }^{25}$

\begin{tabular}{|c|l|}
\hline I & Asymptomatic or minimal headache and slight nuchal rigidity \\
\hline II & Moderate to severe headache, nuchal rigidity, no neurological deficit other than cranial nerve palsy \\
\hline III & Drowsy, confusion or mild focal deficit \\
\hline IV & Stupor, moderate to severe hemiparesis, possible early decerebrate rigidity and vegetative disturbances \\
\hline V & Deep coma, decerebrate rigidity, moribund appearance \\
\hline
\end{tabular}

Appendix A.2. Glasgow Outcome Scale ${ }^{26}$

\begin{tabular}{|c|l|}
\hline 1 & Dead \\
\hline 2 & $\begin{array}{l}\text { Vegetative State } \\
\text { The patient shows no evidence of meaningful responsiveness }\end{array}$ \\
\hline 3 & $\begin{array}{l}\text { Severe Disability } \\
\text { The patient is conscious but needs the assistance of another person for some activities of daily living every day }\end{array}$ \\
\hline 4 & $\begin{array}{l}\text { Moderate Disability } \\
\text { The patient is independent but disabled }\end{array}$ \\
\hline 5 & $\begin{array}{l}\text { Good Recovery } \\
\text { The patient is able to resume normal occupational and social activities, although there may be minor physical or mental deficits }\end{array}$ \\
\hline
\end{tabular}

